\title{
Dual Selection Diversity over Correlated Weibull Fading Channels
}

\author{
Nikos C. Sagias*, George K. Karagiannidis ${ }^{\dagger}$, Dimitris A. Zogas ${ }^{\ddagger}$, P. Takis Mathiopoulos ${ }^{\dagger}$, \\ George S. Tombras*, and Stavros A. Kotsopoulos ${ }^{\ddagger}$ \\ *Laboratory of Electronics, Physics Department, University of Athens, Panepistimiopolis, 15784, Athens, Greece, \\ Email: nsagias@space.noa.gr, gtombras@cc.uoa.gr \\ ${ }^{\dagger}$ Institute for Space Applications and Remote Sensing, National Observatory of Athens, Metaxa \& Vas. Pavlou Street, \\ Palea Penteli, 15236, Athens, Greece, Email: gkarag@space.noa.gr, mathio@space.noa.gr

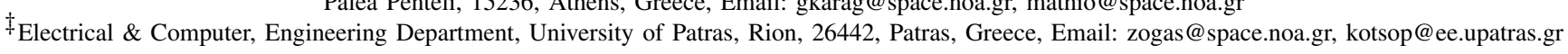

\begin{abstract}
We study the performance of a dual-branch SC receivers over correlated Weibull fading channels. Exact closedform expressions are derived for the probability / cumulative density functions and the moments of the output signal-to-noise ratio (SNR). Important performance criteria, such as average output SNR, amount of fading (AoF), outage probability and average bit error probability (ABEP) for several modulation schemes, are studied and novel closed-form analytical expressions are derived. The proposed analysis is complemented by various performance evaluation results, including the effects of input SNRs unbalancing, fading severity and fading correlation on the overall system performance. Computer simulations results verify the validity and the accuracy of the proposed analysis.
\end{abstract}

\section{INTRODUCTION}

One of the simplest and yet most efficient techniques to overcome the destructive effects of fading in wireless communication systems is diversity. For all diversity techniques, including equal-gain combining (EGC), maximal-ratio combining (MRC), selection combining (SC) and a combination of MRC and SC, referred as generalized-selection combining (GSC) [1], the receiver processes the obtained diversity signals in a fashion that maximizes the systems power efficiency. Among these diversity techniques, SC is the least complicated since the processing is performed only on one of the diversity branches. Traditionally, in SC the combiner chooses the branch with the highest signal-to-noise ratio (SNR), which corresponds to the strongest signal, if equal noise power is assumed among the different branches [1].

The performance of diversity receivers has been extensively studied in the open technical literature for several well-known fading statistical models, such as Rayleigh, Rice, Nakagami-m and Nakagami- $q$, for both independent and correlative fading [1]. Past work concerning the performance of dual-branch diversity receivers with correlative fading can be found in many publications, including [2]-[7]. For example, Malik et al. in [2], presented an efficient approach in analyzing the performance of coherent detection for binary signals with dual-diversity in correlative Rayleigh fading. More recently, Karagiannidis et al. in [3], derived a convergent infinite sum expression for the characteristic function of two correlated Nakagami- $m$ variables and extended the results of [2] to the Nakagami- $m$ fading case. In [4], useful expressions for the outage probability and average bit error probability (ABEP) were presented for a dual selection diversity system with correlated slow Rayleigh and Nakagami- $m$ fading, while in [5], the average output SNR was evaluated. A study of dual MRC and $\mathrm{SC}$ receivers over correlated Rayleigh channels is presented in [6]. Finally in [7], the average output SNR, the amount of fading (AoF) and the outage probability were investigated for dual receivers operating in correlative lognormal fading.

Another fading channel model, namely the Weibull model, has not received as much attention as the above mentioned fading models, despite the fact that it exhibits an excellent fit to experimental fading channel measurements, for indoor [8], [9] and outdoor environments [10], [11]. Only very recently, the topic of communications over Weibull fading channels begun to receive renewed interest. For example, considering the performance of diversity receivers over Weibull fading channels, an analysis for the evaluation of the GSC performance over independent Weibull fading channels was presented in [12]. In this analysis the first two moments and the AoF at the output of the GSC combiner were derived. More recently, in [13], a work related to the second order statistics and the average channel spectral efficiency was presented by Sagias et al. In this work, novel analytical expressions for the average level crossing rate, the average fade duration and the average spectral efficiency, when the Weibull model is considered, was extracted. Two other contributions, dealing with switched [14] and selection diversity [15] in Weibull fading, were also presented. In [14], assuming that the receiver employs switched diversity, expressions for the average SNR, the AoF and the switching rate at the output of the combiner, were derived. Finally, in [15], important performance measures such as the outage probability and the average output SNR were studied, for $L$-branch $S C$ receivers operating in independent Weibull fading environment.

In this paper, we analytically evaluate the performance of a dual-branch SC receiver over correlated Weibull fading channels with arbitrary parameters. Exact closed-form expressions are derived for the probability density function (pdf), the cumulative distribution function (cdf) and the moments of the dual SC output SNR. Capitalizing on these expressions, novel closed-form analytical expressions are derived for the average 
output SNR, the AoF, the outage probability and the ABEP for several modulation schemes. The proposed mathematical analysis is also validated by means of computer simulation.

\section{Statistical Properties of the OUtPut SNR}

\section{A. System and Channel Model}

We consider a dual-branch diversity receiver operating in a Weibull fading environment. The baseband received signal in the $\ell$ th $\left(\ell=1\right.$ and 2) antenna is $z_{\ell}=s r_{\ell}+n_{\ell}$, where $s$ is the transmitted symbol, $r_{\ell}$ is the fading envelope, modelled as a Weibull random variable (rv) and $n_{\ell}$ is the additive white Gaussian noise (AWGN). The usual assumption is made for the AWGN that it is uncorrelated between the two diversity branches and has a double-sided power spectral density $N_{0} / 2$. The Weibull distribution can be regarded as an approximation to the generalized Nakagami distribution of the same order as the Nakagami- $m$ distribution [16] and it's pdf is given by [17]

$$
f_{r_{\ell}}\left(r_{\ell}\right)=\frac{\beta}{\omega_{\ell}}\left(\frac{r_{\ell}}{\omega_{\ell}}\right)^{\beta-1} \exp \left[-\left(\frac{r_{\ell}}{\omega_{\ell}}\right)^{\beta}\right] .
$$

In the above equation, $\omega_{\ell}=\sqrt{\overline{r_{\ell}^{2}} / \Gamma(1+2 / \beta)}, \Gamma(\cdot)$ is the Gamma function [18, eq. (8.310/1)], $\overline{r_{\ell}^{2}}$ is the average signal power and $\beta$ is the Weibull fading parameter $(\beta \geq 0)$. As the value of $\beta$ increases, the severity of the fading decreases. For the special case of $\beta=2$, (1) reduces to the well-known Rayleigh pdf. It is convenient to define the function $d_{\tau}=$ $1+\tau / \beta$, where, in general, $\tau$ is a nonnegative real variable. The corresponding cdf and the moments are given by [17]

$$
F_{r_{\ell}}\left(r_{\ell}\right)=1-\exp \left[-\left(\frac{r_{\ell}}{\omega_{\ell}}\right)^{\beta}\right]
$$

and

$$
E\left\langle r_{\ell}^{n}\right\rangle=\omega_{\ell}^{n} \Gamma\left(d_{n}\right)
$$

respectively, where $E\langle\cdot\rangle$ denotes expectation and $n$ is a positive integer. To model the correlation between the two diversity paths, the bivariate distribution of $r_{1}$ and $r_{2}$ is needed. Among the various distributions belonging to the family of the Weibull bivariate distributions [19], [20], the most suitable distribution to model the correlative fading paths must satisfy the following criteria: i) it's marginal pdfs should be two parameters Weibull distributions, and ii) the range of values for its correlation coefficient should be $[0,1]$. Such a bivariate distribution is derived in [19] as a mixture of its marginals, which are also two parameters Weibull distributions. The complementary cdf (or survival function) of this bivariate rv can be mathematically expressed in the following form [20]

$$
\tilde{F}_{r_{1}, r_{2}}\left(r_{1}, r_{2}\right)=\exp \left\{-\left[\left(\frac{r_{1}}{\omega_{1}}\right)^{\frac{\beta}{\delta}}+\left(\frac{r_{2}}{\omega_{2}}\right)^{\frac{\beta}{\delta}}\right]^{\delta}\right\}
$$

where the dependence factor $\delta(0<\delta \leq 1)$ is related to the correlation coefficient $\left.\rho=\operatorname{cov}\left(r_{1}, r_{2}\right) / \sqrt{\operatorname{var}\left(r_{1}\right) \operatorname{var}\left(r_{2}\right)}\right)$ as

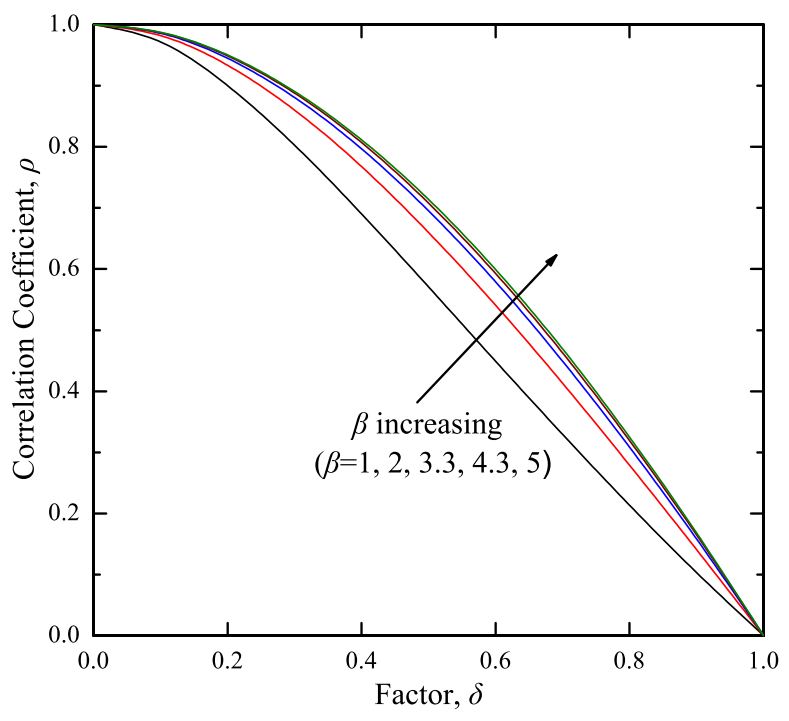

Fig. 1. Correlation coefficient, $\rho$, versus $\delta$, for various values of $\beta$.

follows [19]

$$
\rho=\frac{\Gamma^{2}\left(d_{\delta}\right) \Gamma\left(d_{2}\right)-\Gamma^{2}\left(d_{1}\right) \Gamma\left(d_{2 \delta}\right)}{\Gamma\left(d_{2 \delta}\right)\left[\Gamma\left(d_{2}\right)-\Gamma^{2}\left(d_{1}\right)\right]} .
$$

Since $\rho$ does not directly appear in (4), Fig. 1 plots $\rho$ as a function of $\delta$ for several values of $\beta$. For $\rho=0$ (i.e., $\delta=1$ ), (4) can be expressed as the product of two single Weibull complementary cdfs. Substituting (2) and (4) in [21, eq. (6.22)], the cdf of $r_{1}$ and $r_{2}$ is derived as

$$
\begin{aligned}
F_{r_{1}, r_{2}}\left(r_{1}, r_{2}\right)= & +\exp \left\{-\left[\left(\frac{r_{1}}{\omega_{1}}\right)^{\frac{\beta}{\delta}}+\left(\frac{r_{2}}{\omega_{2}}\right)^{\frac{\beta}{\delta}}\right]^{\delta}\right\} \\
& -\exp \left[-\left(\frac{r_{1}}{\omega_{1}}\right)^{\beta}\right]-\exp \left[-\left(\frac{r_{2}}{\omega_{2}}\right)^{\beta}\right] .
\end{aligned}
$$

Note, that when the diversity input channels are uncorrelated, (6) is also expressed as the product of two single Weibull cdfs.

\section{B. CDF and PDF of the Output SNR}

The instantaneous SNR per symbol for each diversity channel can be expressed as $\zeta_{\ell}=r_{\ell}^{2} E_{s} / N_{0}$, where $E_{s}=E\left\langle s^{2}\right\rangle$ is the transmitted symbols energy. The corresponding average SNR per symbol for each diversity branch is $\bar{\zeta}_{\ell}=\overline{r_{\ell}^{2}} E_{s} / N_{0}=$ $\Gamma\left(d_{2}\right) \omega_{\ell}^{2} E_{s} / N_{0}$. Setting $a=1 / \Gamma\left(d_{2}\right)$, the joint cdf of $\zeta_{1}$ and $\zeta_{2}$ can be obtained directly from (6) as

$$
F_{\zeta_{1}, \zeta_{2}}\left(\zeta_{1}, \zeta_{2}\right)=F_{r_{1}, r_{2}}\left(\omega_{1} \sqrt{\frac{\zeta_{1}}{a \bar{\zeta}_{1}}}, \omega_{2} \sqrt{\frac{\zeta_{2}}{a \bar{\zeta}_{2}}}\right) .
$$

Defining the instantaneous SNR at the SC output as $\zeta_{s c} \triangleq$ $\max \left(\zeta_{1}, \zeta_{2}\right)$ and setting $\zeta_{1}=\zeta_{2}=\zeta_{s c}$ in the above equation, 
the cdf of $\zeta_{s c}$ can be directly obtained as

$$
\begin{aligned}
F_{\zeta_{s c}}\left(\zeta_{s c}\right)= & \exp \left(-D \zeta_{s c}^{\frac{\beta}{2}}\right)-\exp \left[-\left(\frac{\zeta_{s c}}{a \bar{\zeta}_{1}}\right)^{\frac{\beta}{2}}\right] \\
& +1-\exp \left[-\left(\frac{\zeta_{s c}}{a \bar{\zeta}_{2}}\right)^{\frac{\beta}{2}}\right]
\end{aligned}
$$

where $D=a^{-\beta / 2}\left[\bar{\zeta}_{1}^{-\beta /(2 \delta)}+\bar{\zeta}_{2}^{-\beta /(2 \delta)}\right]^{\delta}$. Differentiating (8), the pdf of $\zeta_{s c}$ can be derived as

$$
\begin{aligned}
f_{\zeta_{s c}}\left(\zeta_{s c}\right)= & \frac{\beta}{2}\left\{\frac{1}{a \bar{\zeta}_{1}}\left(\frac{\zeta_{s c}}{a \bar{\zeta}_{1}}\right)^{\frac{\beta}{2}-1} \exp \left[-\left(\frac{\zeta_{s c}}{a \bar{\zeta}_{1}}\right)^{\frac{\beta}{2}}\right]\right. \\
& +\frac{1}{a \bar{\zeta}_{2}}\left(\frac{\zeta_{s c}}{a \bar{\zeta}_{2}}\right)^{\frac{\beta}{2}-1} \exp \left[-\left(\frac{\zeta_{s c}}{a \bar{\zeta}_{2}}\right)^{\frac{\beta}{2}}\right] \\
& \left.-D \zeta_{s c}^{\frac{\beta}{2}-1} \exp \left[-D \zeta_{s c}^{\frac{\beta}{2}}\right]\right\} .
\end{aligned}
$$

\section{Moments of the Output SNR}

By definition, the moments of the output SNR are [21]

$$
E\left\langle\zeta_{s c}^{n}\right\rangle \triangleq \int_{0}^{\infty} \zeta_{s c}^{n} f_{\zeta_{s c}}\left(\zeta_{s c}\right) d \zeta_{s c}
$$

By substituting (9) into (10) and using [18, eq. (3.326/2)], after some straightforward simplifications the $n$th moment of $\zeta_{s c}$ can be derived in a closed-form expression as

$$
E\left\langle\zeta_{s c}^{n}\right\rangle=\left[\bar{\zeta}_{1}^{n}+\bar{\zeta}_{2}^{n}-\left(\bar{\zeta}_{1}^{-\frac{\beta}{2 \delta}}+\bar{\zeta}_{2}^{-\frac{\beta}{2 \delta}}\right)^{-\frac{2 \delta}{\beta} n}\right] \frac{\Gamma\left(d_{2 n}\right)}{\Gamma^{n}\left(d_{2}\right)} .
$$

\section{PERformance AnAlysis}

\section{A. Average Output SNR}

The average output $\operatorname{SNR} \bar{\zeta}_{s c}$ is obtained by setting $n=1$ in (11) as

$$
\bar{\zeta}_{s c}=\bar{\zeta}_{1}+\bar{\zeta}_{2}-\left(\bar{\zeta}_{1}^{-\frac{\beta}{2 \delta}}+\bar{\zeta}_{2}^{-\frac{\beta}{2 \delta}}\right)^{-\frac{2 \delta}{\beta}}
$$

It should be also noted that for independent and identically distributed (i.i.d.) input paths (e.g. $\delta=1$ and $\bar{\zeta}_{1}=\bar{\zeta}_{2}$ ), (12) is identical with that in $[12$, eq. $(8)]$ for the $\operatorname{GSC}(2,1)$ (selecting one out of two input paths).

\section{B. Amount of Fading (AoF)}

Using (11), the AoF of the SC output, defined as the ratio of the variance to the square mean SC output SNR, can be easily expressed in a simple closed-form expression as

$$
\mathrm{AoF} \triangleq \frac{\operatorname{var}\left(\zeta_{s c}\right)}{\bar{\zeta}_{s c}^{2}}=\frac{E\left\langle\zeta_{s c}^{2}\right\rangle}{\bar{\zeta}_{s c}^{2}}-1
$$

It is noted that, for i.i.d. input paths, the expression obtained using (13) for the AoF is the same with [12, eq. (10)] for $\operatorname{GSC}(2,1)$.

\section{Outage Probability}

The outage probability $P_{\text {out }}$ is defined as the probability that the SC output SNR falls below a given threshold $\zeta_{t h}$. Since this probability is simply the probability that neither $\zeta_{1}$ nor $\zeta_{2}$ exceeds $\zeta_{s c}$, the $P_{\text {out }}$ is obtained by replacing $\zeta_{s c}$ with $\zeta_{t h}$ in (8) as

$$
P_{\text {out }}=F_{\zeta_{s c}}\left(\zeta_{t h}\right) .
$$

\section{Average Bit Error Probability (ABEP)}

The most straightforward approach to obtain the ABEP $\bar{P}_{b e}$ is to average the conditional bit error probability $P_{b e}$ over the pdf of the output SNR [1] as

$$
\bar{P}_{b e}=\int_{0}^{\infty} P_{b e}(\zeta) f_{s c}(\zeta) d \zeta
$$

Using well-known expressions for the $P_{b e}$ found in [4], it is easy to realize that for DBPSK and NBFSK (15) requires evaluation of infinite integrals of the form

$$
\Upsilon(\xi)=\int_{0}^{\infty} x^{\frac{\beta}{2}-1} \exp (-x) \exp \left(-\xi x^{\frac{\beta}{2}}\right) d x
$$

Similarly, for $M$-AM, $M$-PSK, $M$-QAM, BFSK and $M$ DPSK, it is required to evaluate finite integrals of the form

$$
\int_{\lambda_{1}}^{\lambda_{2}} \Upsilon[\xi(\varphi)] d \varphi
$$

where the particular values of $\lambda_{1}$ and $\lambda_{2}$ depend upon the modulation scheme. The integral in (16) can be evaluated in closed-form as follows. By expressing the exponential function as a Meijer's G-function [18, eq. (9.301)], i.e., $\exp [-g(x)]=\mathrm{G}_{0,1}^{1,0}\left[\left.g(x)\right|_{0} ^{-}\right][22$, eq. (11)], where $g(\cdot)$ is an arbitrary function, the integral in (16) can be written as

$$
\Upsilon(\xi)=\int_{0}^{\infty} x^{\frac{\beta}{2}-1} \mathrm{G}_{0,1}^{1,0}\left[\left.x\right|_{0} ^{-}\right] \mathrm{G}_{0,1}^{1,0}\left[\left.\xi x^{\frac{\beta}{2}}\right|_{0} ^{-}\right] d x .
$$

Using [22, eq. (21)], the above equation can be expressed in closed-form as

$$
\Upsilon(\xi)=\frac{\left(\frac{k}{l}\right)^{\frac{1}{2}} l^{\frac{\beta}{2}}}{(2 \pi)^{\frac{k+l}{2}}-1} \mathrm{G}_{l, k}^{k, l}\left[\xi^{k} \frac{l^{l}}{k^{k}} \mid \begin{array}{l}
\left\{\frac{2 n-\beta}{2 l}\right\}_{n=1,2 \ldots, l} \\
\left\{\frac{m}{k}\right\}_{m=0,1, \ldots, k-1}
\end{array}\right]
$$

with

$$
\frac{l}{k}=\frac{\beta}{2}
$$

and $k$ and $l$ positive integers. Depending upon the value of $\beta$, a set with minimum values of $k$ and $l$ can be properly chosen in order (20) to be valid (e.g. for $\beta=4.3$ we have to choose $k=20$ and $l=43$ ). For the special case where $\beta$ is an integer, setting $k=2$ and $l=\beta$ (19) reduces to

$$
\Upsilon(\xi)=\sqrt{\frac{2}{\beta}}\left(\frac{\beta}{2 \pi}\right)^{\frac{\beta}{2}} \mathrm{G}_{\beta, 2}^{2, \beta}\left[\frac{\xi^{2}}{4} \beta^{\beta} \mid \begin{array}{c}
\left.\frac{2 n-\beta}{2 \beta}\right\}_{n=1,2, \ldots, \beta} \\
0, \frac{1}{2}
\end{array}\right] .
$$

As an indicative example, the ABEP for DBPSK with integer values of $\beta$ and equal SNRs $\left(\bar{\zeta}_{\ell}=\bar{\zeta}_{0}, \forall \ell\right)$ is expressed as

$$
\bar{P}_{b e}=\frac{\beta}{2\left(a \bar{\zeta}_{0}\right)^{\frac{\beta}{2}}}\left\{\Upsilon\left[\frac{1}{\left(a \bar{\zeta}_{0}\right)^{\frac{\beta}{2}}}\right]-2^{\delta-1} \Upsilon\left[\frac{2^{\delta}}{\left(a \bar{\zeta}_{0}\right)^{\frac{\beta}{2}}}\right]\right\} \text {. }
$$




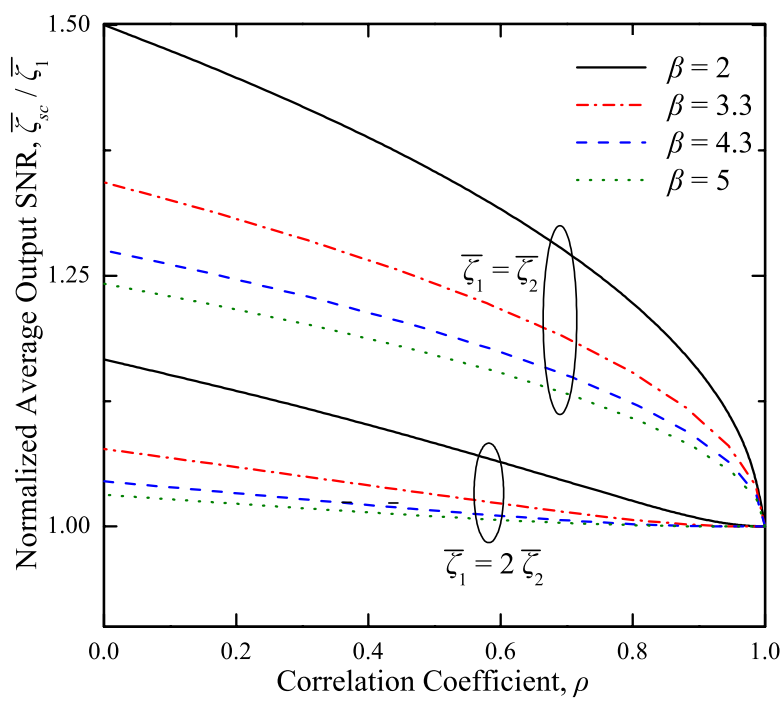

Fig. 2. First branch normalized average output SNR, $\zeta_{s c} / \bar{\zeta}_{1}$, versus the correlation coefficient, $\rho$, for equal $\left(\bar{\zeta}_{1}=\bar{\zeta}_{2}\right)$ and unequal $\left(\bar{\zeta}_{1}=2 \bar{\zeta}_{2}\right)$ input SNRs and for several values of $\beta$.

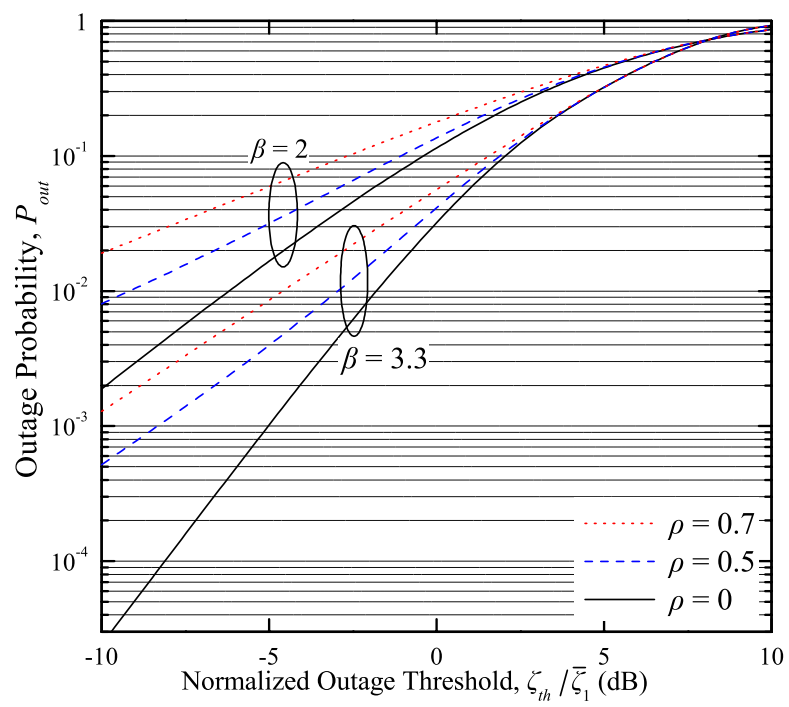

Fig. 3. Outage probability, $P_{\text {out }}$, versus the normalized outage threshold, $\zeta_{t h} / \bar{\zeta}_{1}$, for unequal $\left(\bar{\zeta}_{1}=5 \bar{\zeta}_{2}\right)$ average SNRs for $\rho=0,0.5$ and 0.7 and for $\beta=2$ and 3.3 .

\section{NumericAl RESUlts}

In this section, using the previous mathematical analysis, theoretical results are presented for the performance of dual $\mathrm{SC}$ receivers over correlated Weibull fading channels. In Fig. 2, using (12), the first branch normalized average output SNR, $\zeta_{s c} / \bar{\zeta}_{1}$, is plotted as a function of $\rho$, for equal $\left(\bar{\zeta}_{1}=\bar{\zeta}_{2}\right)$ and unequal $\left(\bar{\zeta}_{1}=2 \bar{\zeta}_{2}\right)$ input branches SNRs and for several values of $\beta$. As expected, the diversity gain decreases as $\rho$ increases. It is interesting to note that, the normalized average output SNR degrades more rapidly as $\rho$ increases, especially for the equal input SNR case and for lower values of $\beta$. For the limiting case of $\rho=0$ the SNR gain of the combiner takes its maximum value, while as $\rho \rightarrow 1$ the corresponding gain

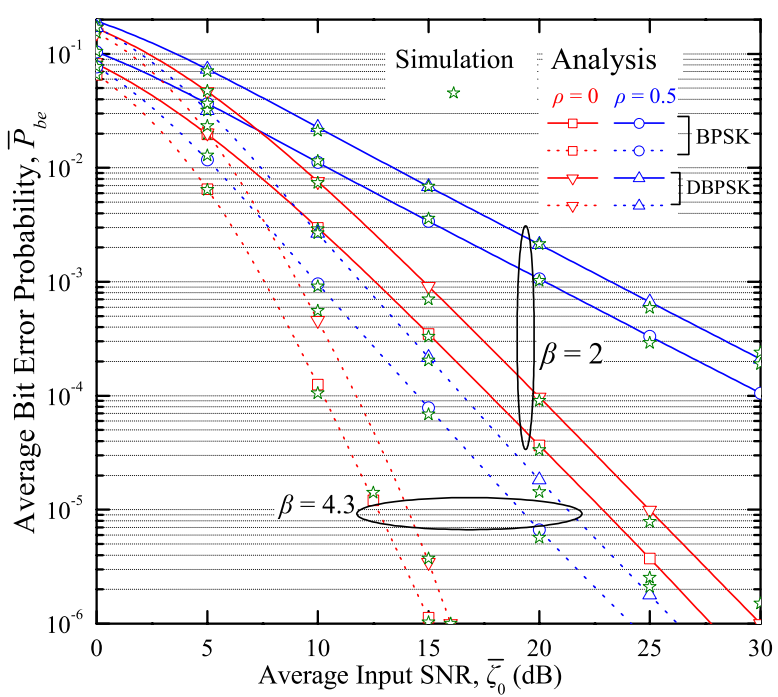

Fig. 4. ABEP of BPSK and DBPSK versus SNR per bit of the first branch, for $\rho=0$ and 0.5 and for $\beta=2$ and 4.3 .

approaches to unity. Additionally, for a fixed $\rho$ the normalized output SNR increases as the severity of fading increases (i.e., as $\beta$ decreases). Similar behavior was observed in [5] where the average SNR of dual SC over correlated Nakagami- $m$ fading channels was studied.

Having numerically evaluated (14), in Fig. 3 the outage probability, $P_{\text {out }}$, performance of the dual SC receiver is presented as a function of the normalized threshold with unequal $\left(\bar{\zeta}_{1}=5 \bar{\zeta}_{2}\right)$ input SNRs and for different values of $\beta$ and $\rho$. For comparison purposes, the curve for $\rho=0$ is also included as a special case for best performance. The obtained results clearly show that the outage performance degrades with an increase of the fading correlation and/or fading severity.

Using (15)-(21), the ABEP of various coherent and noncoherent, binary and multilevel modulation schemes can be obtained. As a typical example, the error performance of BPSK, as a function of the average SNR per bit of the first branch, for $\bar{\zeta}_{\ell}=\bar{\zeta}_{0}$ and for several values of $\beta$ and $\rho$ is illustrated in Fig. 4. The obtained performance evaluation results show that the error performance improves with an increase of $\beta$, while as expected the diversity gain decreases with increasing values of $\rho$. In order to verify these analytical results computer simulations were also performed. For comparison purposes, these results are included in the same figure, verifying the validity of our theoretical approach.

\section{CONCLUSions}

Closed-form expressions for the pdf, the cdf and the moments of the combined SNR at the output of a dual receiver employing SC over both correlated and uncorrelated Weibull fading channels have been derived. Capitalizing on these expressions, important performance criteria, such as average output SNR, AoF, outage probability and ABEP, have been extracted in closed-forms. As an illustration of the mathematical formalism, numerical results of these performance 
criteria were presented, describing their dependence on $\beta$ and $\rho$. Extensive computer simulations have validated the mathematical analysis.

\section{APPENDIX \\ Generation of Correlated Weibull Fading ENVELOPES}

Following the analysis presented in [19], below an efficient algorithm for the generation of two correlated groups of Weibull distributed fading envelopes $r_{1}$ and $r_{2}$, with a joint cdf defined in (6) is given in three steps:

1) Generate five uniform distributed rvs $\left\{U_{n} \in[0,1)\right\}$, $n=1,2,3,4$ and 5 ,

2) Set $U=U_{1}$ and $V=\left\{\begin{array}{l}-\ln \left(U_{2} U_{3}\right), \text { if } U_{5} \leq \delta \\ -\ln \left(U_{4}\right), \text { if } U_{5}>\delta\end{array}\right.$,

3) Set $\left(r_{1}, r_{2}\right)=\left(\omega_{1} U^{\frac{\delta}{\beta}} V^{\frac{1}{\beta}}, \omega_{2}(1-U)^{\frac{\delta}{\beta}} V^{\frac{1}{\beta}}\right)$.

\section{REFERENCES}

[1] M. K. Simon and M.-S. Alouini, Digital Communication over Fading Channels. 1st ed., New York: Wiley, 2000

[2] R. K. Mallik, M. Z. Win, and J. H. Winters, "Performance of dualdiversity EGC in correlated Rayleigh fading with unequal branch SNRs," IEEE Trans. Commun., vol. 50, pp. 1041-1044, July 2002.

[3] G. K. Karagiannidis, D. A. Zogas, and S. A. Kotsopoulos, "BER performance of dual predetection EGC in correlative Nakagami- $m$ fading," to be published in IEEE Trans. Commun.

[4] M. K. Simon and M.-S. Alouini, "A unified performance analysis of digital communications with dual selective combining diversity over correlated Rayleigh and Nakagami- $m$ fading channels," IEEE Trans. Commun., vol. 47, pp. 33-43, Jan. 1999.

[5] Y-K. Ko, M.-S. Alouini, and M. K. Simon, "Average SNR of dual selection combining over correlated Nakagami- $m$ fading channels," IEEE Commun. Lett., vol. 4, pp. 12-14, Jan. 2000.

[6] K. Dietze, C. B. Dietrich, and W. L. Stutzman, "Analysis of a twobranch maximal and selection diversity system with unequal SNRs and correlated inputs for a Rayleigh fading channel," IEEE Trans. Wirel. Commun., vol. 1, no. 1, pp. 274-281, Apr. 2002.
[7] M.-S. Alouini and M. K. Simon, "Dual diversity over correlated lognormal fading channels," IEEE Trans. Commun., vol. 50, pp. 1946-1959, Dec. 2002.

[8] H. Hashemi, "The indoor radio propagation channel," Proc. IEEE, vol 81, pp. 943-968, July, 1993.

[9] F. Babich and G. Lombardi, "Statistical analysis and characterization of the indoor propagation channel," IEEE Trans. Commun., vol. 48, pp. 455-464, Mar. 2000.

[10] N. S. Adawi, et al., "Coverage prediction for mobile radio systems operating in the $800 / 900 \mathrm{MHz}$ frequency range," IEEE Trans. Veh. Technol., vol. 37, no. 1, pp. 3-72, Feb. 1988.

[11] N. H. Shepherd, "Radio wave loss deviation and shadow loss at 900 MHz," IEEE Trans. Veh. Technol., vol. 26, pp. 309-313, 1977.

[12] M.-S. Alouini and M. K. Simon, "Performance of generalized selection combining over Weibull fading channels," in Proc. IEEE VTC'01, May 2001, Rhodes, Greece, pp. 1735-1739.

[13] N. C. Sagias, D. A. Zogas, G. K. Karagiannidis, and G. S. Tombras, "Channel capacity and second order statistics in Weibull fading," to be published in IEEE Commun. Lett.

[14] N. C. Sagias, D. A. Zogas, G. K. Karagiannidis, and G. S. Tombras, "Performance analysis of switched diversity receivers in Weibull fading," Electron. Lett., vol. 39, no. 20, pp. 1472-1474, Oct. 2003.

[15] N. C. Sagias, P. T. Mathiopoulos, and G. S. Tombras, "Selection diversity receivers in Weibull fading: Outage probability and average signal-tonoise ratio," Electron. Lett., Dec. 2003.

[16] M. A. Taneda, J. Takada, and K. Araki, "The problem of the fading model selection," IEICE Trans. Commun., vol. E84-B, no. 3, pp. 355358, Mar. 2001.

[17] K. Bury, Statistical Distributions in Engineering. Cambridge University Press, 1999.

[18] I. S. Gradshteyn and I. M. Ryzhik, Table of Integrals, Series, and Products. 5th ed., New York: Academic, 1994.

[19] J. C. Lu and G. K. Bhattaacharyya, "Some new constructions of bivariate Weibull models," Annals of Inst. of Stat. Math., vol. 42, no. 3, pp. 543559,1990

[20] S. Kotz, N. Balakrishnan, and N. L. Johnson, Continuous Multivariate Distributions. Volume 1: Models and Applications. Wiley, 2000.

[21] A. Papoulis, Probability, Random Variables, and Stochastic Procceses. 3rd ed., McGraw-Hill.

[22] V. S. Adamchik and O. I. Marichev, "The algorithm for calculating integrals of hypergeometric type functions and its realization in REDUCE system," in Proc. International Conf. on Symbolic and Algebraic Computation, 1990, Tokyo, Japan, pp. 212-224. 